\title{
Interstellar Pathfinder - A Mission to the Inner Edge of the Interstellar Medium
}

\author{
D.J. McComas ${ }^{1}$, P.A. Bochsler ${ }^{2}$, L.A. Fisk ${ }^{3}$, H.O. Funsten ${ }^{4}$, J. Geiss ${ }^{5}$, \\ G. Gloeckler ${ }^{3,6}$, M. Gruntman ${ }^{7}$, D.L. Judge ${ }^{7}$, S.M. Krimigis ${ }^{8}$, R.P. Lin ${ }^{9}$, \\ S. Livi ${ }^{8}$, D.G. Mitchell ${ }^{8}$, E. Möbius ${ }^{10}$, E.C. Roelof ${ }^{8}$, \\ N.A. Schwadron ${ }^{1}$, M. Witte ${ }^{11}$, J. Woch ${ }^{11}$, P. Wurz ${ }^{2}$, T.H. Zurbuchen ${ }^{3}$ \\ ${ }^{1}$ Southwest Research Institute, San Antonio, TX USA \\ ${ }^{2}$ University of Bern, Bern, Switzerland \\ ${ }^{3}$ University of Michigan, Ann Arbor, MI USA \\ ${ }^{4}$ Los Alamos National Laboratory, Los Alamos NM USA \\ ${ }^{5}$ International Space Science Institute, Bern, Switzerland \\ ${ }^{6}$ University of Maryland, College Park, MD USA \\ ${ }^{7}$ University of Southern California, Los Angeles, CA USA \\ ${ }^{8}$ The Johns Hopkins University, Laurel, MD USA \\ ${ }^{9}$ University of California Berkeley, Berkeley, CA USA \\ ${ }^{10}$ University of New Hampshire, Durham, NH USA \\ ${ }^{11}$ Max Planck Institut für Aeronomie, Lindau, Germany
}

\begin{abstract}
Interstellar Pathfinder (ISP), our first step into the interstellar medium, is a scientific investigation to study the outer boundary of our heliosphere and the interstellar matter that flows into it. A wind of interstellar neutral gas penetrates to within several astronomical units (AU) of the Sun, giving us a direct sample of present-day galactic matter. ISP is a mission to this inner edge of the interstellar medium. Using highly sensitive instrumentation, ISP will determine the composition of our local interstellar environment. It will also take the first global images of the boundary region of the heliosphere at 100 to $150 \mathrm{AU}$. These measurements will allow ISP to answer fundamental questions about the origin of the solar system and the stars, about the evolution of our galaxy and of the universe, and about the characteristics of our local galactic environment and its influence on the heliosphere.
\end{abstract}

\section{Scientific Background and Goals}

As the heliosphere moves through the local interstellar medium, it is thought to form several plasma structures including an upstream bow shock, a heliopause that separates the solar wind from the ionized portion of the interstellar medium at about 150 astronomical units (AU), and a termination shock of the supersonic flow of the solar wind at $\sim 100$ AU. This interaction is shown schematically in the left panel of Figure 1. Because these structures are so distant, the common conception is that only distant
Voyager, or eventually Interstellar Probe, has any expectation of reaching interstellar space. However, the inner edge of the interstellar medium is far closer. The solar wind does not stop neutral particles from the interstellar medium from penetrating relatively undisturbed to within 3 AU of the Sun. Further, energetic neutral atoms generated from ions accelerated in and around the various structures in the outer heliosphere also propagate freely into the inner heliosphere. 
Interstellar Pathfinder (ISP) is a mission to this inner edge of interstellar space. The right side of Figure 1 shows a blowup of the inner heliosphere, the ISP orbit, and the various sources observed by this mission. ISP will carry instruments that are much only accessible sample of the present-day galaxy. By comparing with the known composition of our solar system, which formed from galactic matter 4.6 billion years ago, we can see how galactic material evolved chemically over time, extrapolate what it was like in the early universe, and unravel the dynamical

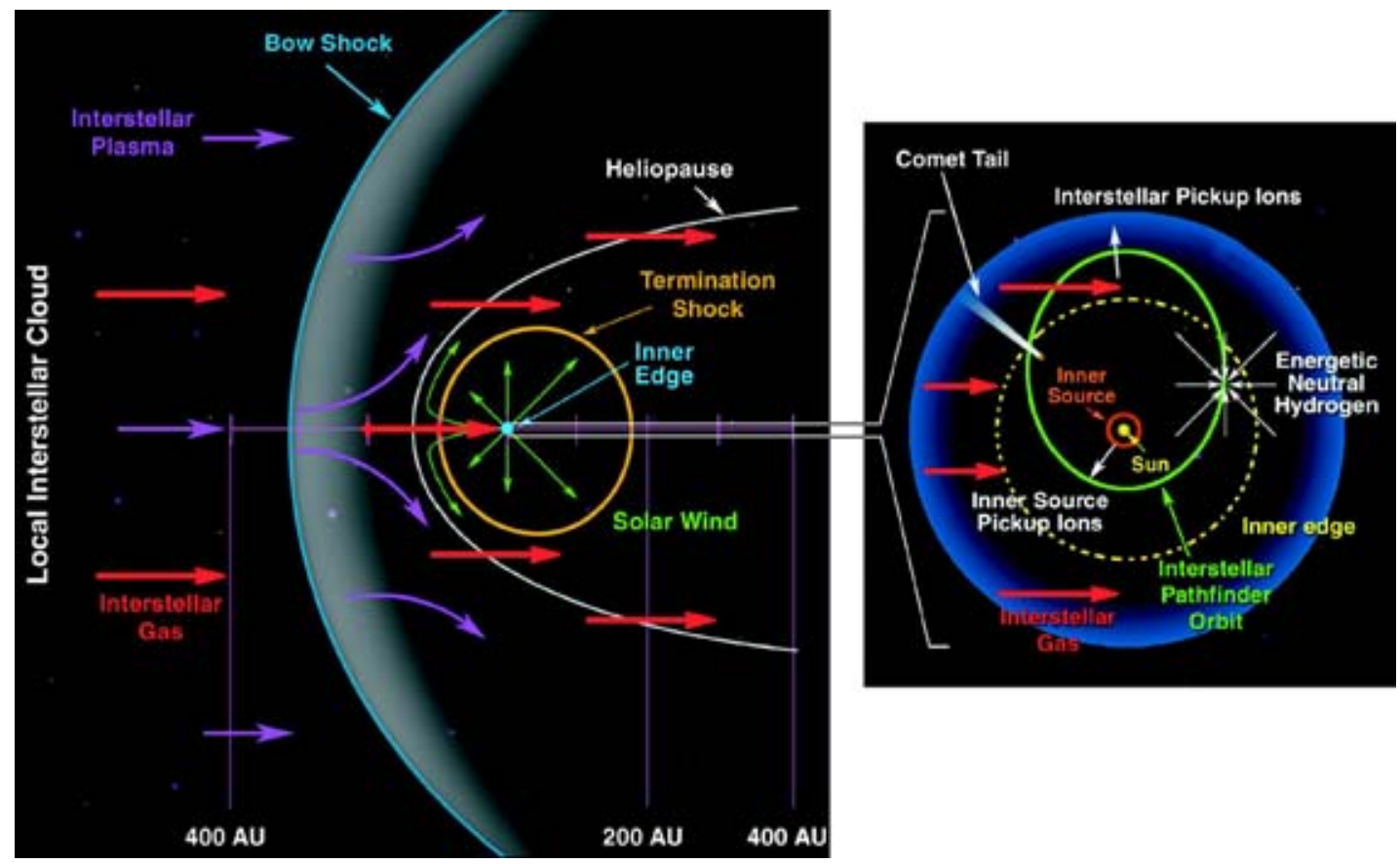

Figure 1. Schematic representation of the heliospheric structure formed by its interaction with the local interstellar medium (left) and an expanded view of the processes observable within the region of space sampled by Interstellar Pathfinder (right).

more capable than anything previously flown, allowing us to determine the properties and composition of the neutral interstellar gas, including its isotopic composition, and the structure of the outer heliosphere, for the first time. These measurements will reveal vital new data on the physical state and composition of

the interstellar medium and our heliosphere's interaction with it.

Interstellar Pathfinder was designed to answer five fundamental questions:

1. What is the composition of the interstellar medium, and what does this tell us about the evolution of the universe and galaxy and about the birthplace of the Sun? The elemental and isotopic composition of cosmic matter gives crucial information needed to determine the origin and evolution of the universe. The Pickup Ion Composition Spectrometer (PICSPEC) will gather this information for the local interstellar cloud, the processes occurring in our galaxy, such as the rate of in falling matter and stellar nucleosynthesis, and place limits on the location where our Sun was born.

2. What is the physical state of the local interstellar cloud, and how does the heliosphere interact with it? The Neutral Interstellar Helium Detector (NIHED) will directly determine the flow speed, temperature, and density of interstellar helium neutrals, while PICSPEC provides the complementary information for hydrogen from pickup ions. This combination reveals the physical characteristics of the surrounding interstellar cloud and its dynamic interaction with the heliosphere.

3. What are the characteristics of the heliospheric termination shock? The Shock and Heliosphere Energetic Neutral Atom (SHENA) imager will provide a full-sky map of the energetic processes at the termination shock where the solar wind is slowed to subsonic speed. These images will show us the nature and spatial variation of this shock. When we combine them with data from the Voyager Spacecraft, which 
will pass through this region soon, we will be able to formulate a complete global picture of this important, distant boundary.

4. What is the nature of the inner source of pickup ions and the composition of comets? The inner source of pickup ions is created when the solar wind is embedded in dust grains and released as neutrals to be ionized and picked up by the flow of the solar wind. Superimposed on the inner source, and offering a significant opportunity for discovery, will be pickup ions in the distant tails of comets. If a sufficient number of comets are detected, a systematic study of the volatile elements in comets will be possible, particularly in small comets.

5. What is the nature of the interaction of pickup ions with the solar wind? The interaction of solar wind with the interstellar neutrals is brought about through pickup ions. The PICSPEC instrument is the first of its kind, designed specifically for the study of pickup ions. The dynamic behavior of these pickup ions will be studied in unprecedented detail.

\section{IMPLEMENTATION}

All of the science goals of the Interstellar Pathfinder mission can be accomplished with a suite of three primary instruments and two supporting "off-theshelf" monitors:

- The Pickup Ion Composition Spectrometer (PICSPEC) is a large-collecting-power ion mass spectrometer, with a collecting power of over 300 times that of the Ulysses SWICS instrument. SWICS provided data that enabled the discovery of most of the pickup ion species and led to the dramatic increase in our present understanding of the LIC. For PICSPEC, we achieve this high sensitivity by eliminating the need to step in voltage, as was done on SWICS. Rather, the deflection is measured by sensing the positions of the particles. This simple but powerful change permits us to measure with unprecedented sensitivity the velocity distributions and abundance of all pickup ions, including the rare isotopes $\mathrm{D},{ }^{3} \mathrm{He},{ }^{18} \mathrm{O},{ }^{22} \mathrm{Ne}$ and $38 \mathrm{Ar}$, as well as molecular pickup ions up to mass 50.

- The Shock and Heliosphere Energetic Neutral Atom (SHENA) imager is a full sky imager of energetic ENAs coming from the termination shock. SHENA is optimized for measuring fluxes of heliospheric energetic $\mathrm{H}$ atoms and has the required large geometric factor and angular resolution to accumulate $7^{\circ}$ pixel resolution images of the heliospheric termination shock in five energy bands. SHENA measures neutral $\mathrm{H}$ atoms in the 0.3-6.0 $\mathrm{keV}$ energy range by first converting them to $\mathrm{H}^{+}$, using foil stripping, and then using energy and coincidence analysis of the post-accelerated ions. This technique combines four simple, flight-proven elements into a new configuration for detecting and imaging ENAs.

- The Neutral Interstellar Helium Detector is an imager of interstellar neutral $\mathrm{He}$, as well as of $\mathrm{O}$ and Ne during favorable portions of the orbit. NIHED is a high-sensitivity, high-angular-resolution version of Ulysses/GAS instrument, with 400 times larger collecting area and a far better signal-to-noise than GAS. NIHED has the necessary sensitivity, angular resolution, and background rejection to measure precisely the interstellar neutral $\mathrm{He}$ bulk flow parameters (flow vector, temperature, density), detect nonthermal tails in the He distributions, and determine the flow speed of interstellar $\mathrm{O}$ and $\mathrm{Ne}$ atoms.

- The Solar Wind Monitor comprises the solar wind ion and 3D electron spectrometers.

- The Solar Ultraviolet sensor provides a monitor of solar extreme ultraviolet.

The principal requirement for ISP's orbit is to reach the inner edge of interstellar space at $\sim 3 \mathrm{AU}$ from the Sun. Thus, a 3-year, in-ecliptic, 1 x 3 AU heliocentric orbit is used. This orbit places the spacecraft within the inner edge of the neutral LIC for more than 1 year, which is necessary for collecting the very rare interstellar isotopes. It is also ideal for accumulating detailed ENA images of the termination shock and the vast region beyond. Additional 3-year orbits are possible, but are not required to fulfill the primary objectives of the ISP mission.

\section{STATUS AND CONCLUSIONS}

Interstellar Pathfinder was rated Category 1 (the top science rating) and low risk in the 2001 Medium Explorer (MIDEX) competition, but for some unknown reason was not selected for Phase A study and mission development. Since then, the Sun-Earth Connection community has been carrying out two major strategic planning exercises. Both of these, NASA's Sun-Earth Connection Roadmap and the National Research Council's Decadal Survey, have rated the ISP science as extremely high priority, further solidifying the case for this critical mission. The Interstellar Pathfinder team will continue to 
optimize our mission design and implementation plan and, with the support of the heliospheric community, expect to be an outstanding contender for finally bringing a MIDEX mission to heliospheric science.

\section{ACKNOWLEDGMENTS}

We gratefully acknowledge all of the important contributions made to the Interstellar Pathfinder mission proposals by the host of scientists, engineers, and technical and administrative support personnel, who have worked on this effort over the years. 